\title{
Editorial
}

\section{Twin Gestation and Premature Birth}

\section{John Morrison, MD}

Journal of Perinatology (2005) 25, 1-3. doi:10.1038/sj.jp.7211224

Preterm birth before 37 weeks' gestation is the most common catastrophic, and costly complication of pregnancy. In this country, the incidence of preterm birth has risen from 9\% in 1981 to above $11 \%$ in $2002 .{ }^{1}$ Prematurity remains the leading cause of neonatal morbidity and mortality, accounting for 60 to $80 \%$ of deaths in live born infants without congenital malformations. Up to $10 \%$ of surviving newborns at very low birth weight $(<1500 \mathrm{~g})$ will develop mental retardation, visual disabilities, or neurobehavioral dysfunction. An average of 10.2 billion dollars per year is spent for newborn care and while $11 \%$ of deliveries are preterm, a disproportionate 6 billion dollars (57\%) is spent to care for these infants. ${ }^{2}$ In twin gestations the problem increases logarithmically as these pregnancies deliver earlier than singleton gestations and there are two infants to contribute to excess morbidity and cost. For example, in one study singleton pregnancies averaged $\$ 9800$ per birth but the cost of twin births were almost $\$ 38,000$. $^{3}$ Another recent investigation demonstrated that $58 \%$ of twins delivered before 37 weeks and $11.9 \%$ before 34 weeks. ${ }^{4}$ Births before 32 weeks represent only 1 to $2 \%$ of overall deliveries but account for $50 \%$ of all the long-term neurologic morbidity and $60 \%$ of the neonatal mortality. Previous studies have noted that most of the serious neonatal morbidity occurred at $<1500 \mathrm{~g}$ and therefore, when 32 to 34 weeks' gestation was attained, few clinicians use aggressive treatment to forestall delivery. ${ }^{5}$ The majority of twin pregnancies now deliver at 34 to 36 weeks' gestation. More recently, however, attention has been focused on treatable conditions in near-term pregnancies (33 to 37 weeks) as clinical management of twin gestations is often challenging at all stages of pregnancy. Near term, when the highest risk of severe morbidity and mortality has passed, the clinician may feel safe with delivery occurring at this time, although there are no data to support discretionary preterm birth.

In the current study, Elliott et al. ${ }^{6}$ investigated pregnancy outcome in 3252 twin gestations (6504 infants) who had been

Department of Obstetrics and Gynecology, Department of Pediatrics, University of Mississippi School of Medicine, Jackson, MS, USA.

Address correspondence to John Morrison, MD, Professor, Department of Obstetrics and Gynecology, Department of Pediatrics, University of Mississippi School of Medicine, OB/GYN, 2500 N. State Street, Jackson, MS 39216, USA. receiving outpatient management with uterine monitoring and daily telephonic nursing assessment who delivered between 33.0 and 36.9 weeks' gestation. The majority (78\%) of deliveries near term were identified as being indicated due to preterm labor or early PROM or having maternal/fetal factors necessitating delivery, although $22 \%$ of deliveries were discretionary: elective inductions or scheduled cesarean deliveries without maternal or fetal indications. This latter group of women had an increase in cesarean births (36.8\%) and almost $40 \%$ of infants required NICU admission. This study also showed that with each advancing week of gestation, birth weight increased while neonatal length of stay, NICU admission rate, and utilization of mechanical ventilation decreased $(p<0.001)$. For example, there was a $44 \%$ decrease in NICU admissions when infants with discretionary delivery at 34 weeks were compared to those at 36 weeks ( 65.2 versus $21.2 \%$, respectively, $p<0.001)$. This article underscores one of the two critical caveats regarding the management of twin pregnancy; discretionary deliveries are ill advised between 33.0 and 36.9 weeks' gestation.

The other very important aspect of clinical management for twin gestations is to forestall deliveries at $<32$ weeks; a complex task. To begin with, early recognition of multifetal pregnancy is critical as well as documentation of chorionicity and confirmation of gestational age for cogent management. ${ }^{7}$ Secondly, serial sonographic cervical examination at 14 to 20 weeks' gestation is also recommended as it has been shown that many patients with subtle cervical change (singleton and twins) had regular uterine contractions. ${ }^{8}$ Particularly, in twins, this may account for the increased percentage of late second trimester and early third trimester spontaneous births. Early pregnancy assessment also provides opportunity for systematic preterm birth prevention management strategies to be employed. One such comprehensive management system involves monitoring for uterine contractions 2 hours per day and daily conversation with a dedicated perinatal nurse to detect changes in patients' signs or symptoms. This management plan has been extensively reported in the literature, and 52 of 61 peer-reviewed studies have shown effectiveness in significantly prolonging gestation by allowing an earlier diagnosis of preterm labor leading to more effective tocolysis which results in a reduction in preterm deliveries, NICU admissions, and neonatal cost. $^{9}$ Among studies reporting on multifetal pregnancies, 15 of 19 investigations confirmed similar positive findings. It is important to emphasize that daily contact with a dedicated perinatal nurse, monitoring contractions objectively, and having access to 24 hour per day/7 day per week emergency nursing contact are critical in making this system work efficiently. For example, as shown by 
Dyson et al., ${ }^{10,11}$ if the nurses are not dedicated only to such a comprehensive system, or if an inappropriate monitor is used, results can change from a positive effect on prevention of preterm birth to no noticeable difference. In addition, if there is no perinatal nursing service, or if the physicians cannot use the uterine activity clinically, preterm births before 32 to 34 weeks will not be reduced. For example, in one multicenter study, 254 women at high risk and 52 women at low risk for preterm birth had uterine contraction monitoring for 2 hours per day and the authors noted an increased contraction frequency for patients delivering at $<35$ weeks' gestation compared to women delivering at term. ${ }^{12}$ However, physicians were blinded to the uterine activity data and no perinatal nursing service was utilized. Inexplicably, the authors concluded that monitoring uterine activity was not helpful to clinicians in preventing preterm birth. In contrast, Elliott, Lam, and others have published many articles showing that a comprehensive management system including home electronic contraction monitoring works well (particularly when aggressive tocolytic therapy is employed in those developing preterm labor) in singleton and twin gestations if all system components are present. ${ }^{9}$

Back to the other end of the gestational age spectrum, the clinical findings in the current paper by Elliott agree with other studies in the literature regarding the benefit of avoiding discretionary delivery. One investigator noted that there had been a $14 \%$ increase in preterm labor induction between 34 and 36 weeks in twin gestation. ${ }^{13}$ In another large study 8000 twin pairs over a 10 -year period of time were studied and significantly higher perinatal mortality/morbidity rates were noted at $\leq 35$ weeks' gestation. ${ }^{14}$ Büscher also found a difference in the birth weight for neonates in twin deliveries delivered at $>37$ weeks' gestation versus those who delivered between 34.0 and 36.9 weeks, with nearly $70 \%$ of twin gestations delivering at 34.0 to 36.9 weeks being admitted to the NICU. ${ }^{15}$ Other studies have well documented the link between gestational age at delivery and NICU admission as well as nursery length of stay.

In summary, it appears that twin gestations achieve the best preterm birth rate reduction by early confirmation of gestational age and chorionicity, serial cervical length assessment, as well as, comprehensive preterm birth prevention management, and excellent clinical antepartum assessment, all of which enables such gestations to have the greatest chance of attaining a gestational age of at least 33 weeks. The message by Elliott et al., in this issue of The Journal is for the obstetrician to be wary of instituting or failing to avoid delivery of twin gestations between 33.0 and 36.9 weeks' gestation when it is not truly indicated. One must remember that although neonatal morbidity and higher health care costs occur with delivery at $<32$ weeks' gestation, that this group comprises the vast minority of gestations, as upwards of $95 \%$ of preterm deliveries occur between 33 and 37 weeks' gestation.
Obviously, if there are fetal/maternal indications for delivery, physician judgment is needed to determine whether the baby is better off in utero or in the nursery. On the other hand, physicianallowed or discretionary deliveries between 33.0 and 36.9 weeks' gestation should be abandoned because they correspond to an increase in NICU admissions and hospital days, thus unnecessarily expanding neonatal morbidity as well as cost. Therefore, perhaps it is time for an old dog to learn new tricks. In the past, I had taught my residents that the battle had been won if we could get pregnancies to 34 weeks. ${ }^{16}$ Although a desirable achievement, reaching 34 weeks does not entitle the physician to ignore the consequences of near-term delivery. Without indications for delivery we are best advised to use all our clinical skills to continue the gestation, as nature intended, to at least 36 to 37 weeks in utero.

\section{References}

1. Goldenberg RL. The management of preterm labor. Obstet Gynecol 2002;100:1020-37.

2. St John EB, Nelson KG, Oliver SP, Bishnoi RR, Goldenberg RL. Cost of neonatal care according to gestational age at birth and survival status. Am J Obstet Gynecol 2000;182:170-5.

3. Callahan TL, Hall JE, Ettner SL, Christiansen CL, Greene MF, Crowley Jr WF. The economic impact of multiple-gestation pregnancies and the contribution of assisted-reproduction techniques to their incidence. N Engl J Med 1994;331(4):270-1.

4. Joseph KS, Allen AC, Dodds L, Vincer MJ, Armson BA. Causes and consequences of recent increases in preterm birth among twins. Obstet Gynecol 2001;98:57-64.

5. Ananth CV, Joseph KS, Smulian JC. Trends in twin neonatal mortality rates in the United States, 1989 through 1999: influence of birth registration and obstetric intervention. Am J Obstet Gynecol 2004;190: $1313-21$.

6. Elliott JP, Istwan NB, Collins A, Rhea D, Stanziano G. Indicated and nonindicated preterm delivery in twin gestations: impact on neonatal outcome and cost. Journal of Perinatol 2005.

7. Dalton ME, Goldman CJ, Morgan MA, Robinson JN. Multiple pregnancy: knowledge and practice patterns of obstetricians and gynecologists. PubMed 2004;104(2):232-7.

8. Berghella V, Talucci M, Desai A. Does transvaginal sonographic measurement of cervical length before 14 weeks predict preterm delivery in high risk pregnancies? Ultrasound Obstet Gynaecol 2003;21(2): $140-4$.

9. Morrison JC, Chauhan SP. Current status of home uterine activity monitoring. Clin Perinatol 2003;30:757-801.

10. Dyson DC, Crites YM, Ray DA, Armstrong MA. Prevention of preterm birth in high-risk patients: the role of education and provider contact versus home uterine monitoring. Am J Obstet Gynecol 1991;164:756-62.

11. Dyson DC, Danbe KH, Bamber JA, et al. Monitoring women at risk for preterm labor. N Engl J Med 1998;338:15-9.

12. Iams JD, Newman RB, Thom EA, et al. Frequency of uterine contractions and the risk of spontaneous preterm delivery. N Engl J Med 2002;346: $250-5$ 
13. Joseph KS, Allen AC, Dodds L, Vincer MJ, Armson BA. Causes and consequences of recent increases in preterm birth among twins. Obstet Gynecol 2001;98:57-64.

14. Hartley RS, Emanuel I, Hitti J. Perinatal mortality and neonatal morbidity ranges among twin pairs at different gestational ages: optimal delivery timing at 37 to 38 weeks' gestation. Am J Obstet Gynecol 2001;184:451-8.
15. Büscher U, Horstkamp B, Wessel J, Chen FCK, Dudenhausen JW. Frequency and significance of preterm delivery in twin pregnancies. Int J Gynaecol Obstet 2000;69:1-7.

16. Fox MD, Allbert JR, McCaul JF, Martin RW, McLaughlin BN, Morrison JC. Neonatal morbidity between 34 weeks' gestation. J Perinatol 1993;XIII:349-53. 